\title{
Improved Internal Model Control Technique for Position Control of AC Servo Motors
}

\author{
Abdul Wali Abdul Ali ${ }^{1 *}$, Abdullah Hadi Yahya Alquhali ${ }^{2}$ \\ ${ }^{1}$ Centre for Electric Energy and Automation (CEEA) Faculty of Engineering, Multimedia University, Cyberjaya, Malaysia \\ ${ }^{2}$ Centre for Wireless Technology (CWT), Faculty of Engineering, Multimedia University, Cyberjaya, Malaysia. \\ *Corresponding author: walikdr17@gmail.com, Tel: 601-114320521, Tel: 601-123442394
}

\begin{abstract}
This paper focuses on the simulation analysis of the conventional Internal Model Control (IMC) technique and the development of two proposed control techniques for the position control of AC Servo Motor. Internal Model Control (IMC) technique [1] was only able to control the AC Servo Motor under static load condition. Also, it had step response problems, and it was not robust against external disturbances. For these reasons, the IMC technique was further improved to control the AC Servo Motor under dynamic load conditions by proposing Amended Internal Model Controller (AIMC). The step response and the robustness of AIMC against external disturbances were further improved by proposing AIMC+FLC. Where a Fuzzy Logic Controller (FLC) is designed and connected with the AIMC.
\end{abstract}

Keywords: AC Servo Motor, AIMC, AIMC+FLC, Control Stability, Bode diagram, Dynamic load, External disturbances, IMC, Static load, Step response.

(C) 2020 Penerbit UTM Press. All rights reserved

Article History: received 15 October 2019; accepted 24 March 2020; published 25 April 2020

\section{INTRODUCTION}

AC Servo Motors are widely used for the precise control of dynamic loads. Many control techniques consisting of advanced hardware and Sensors were used to achieve the objectives. However, many internal and external factors have an impact on the precision of control for the AC Servo Motor. The precise control of position, speed, and torque were the main problems on AC Servo Motor. Such Motors were extremely demanded in the various control applications, i.e., Process/industrial control, medical equipment, Computer Numerical Control machines, conveying, robotics, textiles, Liquid Cristal Display manufacturing and etc. It was highly desired to have precise responses for the position tracking and have a fast recovery for the dynamic loads or external interference [2]. The Internal Model Control (IMC) technique offered high precision control for the AC Servo Motor, it was simple and low cost, and it could be improved for the precise operation. This control strategy was designed for the control of the AC Servo Motor when the load connected to the motor was stationary, or it did not take the external disturbances into account. To meet the industrial needs of controlling the dynamic loads with precision; in this paper, the AC Servo Motor position control technique using the IMC strategy [2] was further utilized for the precise control of the position of the $\mathrm{AC}$ Servo Motor when the load connected to the motor was dynamic. This amended control method was designed while taking the external disturbances into account, and it was named as Amended Internal Model Control (AIMC). The step response and robustness of the AIMC against external disturbances were further improved by designing a Fuzzy Logic Controller (FLC) and connecting it with the AIMC. This proposed control system was called as AIMC+FLC.

\section{MODELING OF THE AC SERVO MOTOR}

The system model contained two-phase AC Servo Motor and an inertia load attached at the output shaft of the motor [3]. The dynamic equation for the mechanical system was as defined by (1).

$$
T_{C}=T_{L}+B_{M} \theta(t)+J_{M} \ddot{\theta}(t)
$$

Where

$T_{C}$ was the control torque $(\mathrm{Nm})$

$T_{L}$ was the torque of the mechanical load $(\mathrm{Nm})$

$B_{M}$ was the friction coefficient of the motor

$\theta$ was the angular position of the motor ( $\mathrm{rad})$

$J_{M}$ was the moment of inertia of the motor $\left(\mathrm{Kg} \cdot \mathrm{m}^{2}\right)$

$\ddot{\theta}$ was the angular acceleration of the motor $\left(\frac{\mathrm{rad}}{\mathrm{s}^{2}}\right)$

The control torque of the AC Servo Motor was given by

Where

$$
T_{C}=K_{1} V(t)-K_{2} \dot{\theta}(t)
$$

$K_{1}$ was the motor constant $\left(\frac{\mathrm{Nm}}{V}\right)$

$V$ was the input rated voltage $(V)$

$K_{2}$ was the motor constant $\left(\frac{\mathrm{Nm} / \mathrm{rad}}{\mathrm{s}}\right)$

$\dot{\theta}$ was the angular velocity of the motor $\left(\frac{\mathrm{rad}}{\mathrm{s}}\right)$

The mechanical load torque was given by 


$$
T_{L}=B_{L} \theta(t)+J_{L} \ddot{\theta}(t)
$$

Where

$B_{L}$ was the friction coefficient of the load $J_{L} \quad$ was the moment of inertia of the load $\left(\mathrm{Kg} \cdot \mathrm{m}^{2}\right)$

By equating (1) and (2), the resulting equation became as (4)

$$
T_{L}+B_{M} \theta(t)+J_{M} \ddot{\theta}(t)=K_{1} V(t)-K_{2} \dot{\theta}(t)
$$

By substituting (3) into (4)

$B_{L} \theta(t)+J_{L} \ddot{\theta}(t)+B_{M} \theta(t)+J_{M} \ddot{\theta}(t)=K_{1} V(t)-$ $K_{2} \dot{\theta}(t)$

By taking Laplace Transform on (5), the resulting equation became as (6)

$B_{L} s \theta(s)+j_{L} s^{2} \theta(s)+B_{M} s \theta(s)+J_{M} s^{2} \theta(s)=$ $K_{1} V(s)-K_{2} s \theta(s)$

By modifying (6), the resulting equation became as (7)

$K_{1} V(s)=B_{L} s \theta(s)+j_{L} s^{2} \theta(s)+B_{M} s \theta(s)+$ $J_{M} s^{2} \theta(s)+K_{2} s \theta(s)$

The motor transfer function was obtained between $\theta(s)$ and $V(s)$

$$
K_{1} V(s)=\left(J_{M} s^{2}+J_{L} s^{2}+B_{M} s+B_{L} s+K_{2} s\right) \theta(s)
$$

By modifying (8)

$\frac{\theta(s)}{V(s)}=\frac{K_{1}}{\left(J_{M}+J_{L}\right) s^{2}+\left(B_{M}+B_{L}+K_{2}\right) s}$

Equation (9) could be further modified as below

$$
\frac{\theta(s)}{V(s)}=\frac{K_{1}}{s\left[\left(B_{M}+B_{L}+K_{2}\right)\left(\frac{\left(J_{M}+J_{L}\right) s}{B_{M}+B_{L}+K_{2}}+1\right)\right]}
$$

The system's gain and time parameters were as shown below the system's gain parameter

$$
\begin{aligned}
& \text { System's gain parameter }=K_{P}=\frac{K_{1}}{B_{M}+B_{L}+K_{2}} \\
& \text { System's time parameter }=\tau_{P}=\frac{J_{M}+J_{L}}{B_{M}+B_{L}+K_{2}}
\end{aligned}
$$

The model of the AC Servo Motor was obtained by substituting (11) and (12) into (10), as shown in (13).

$$
\frac{\theta(s)}{V(s)}=G_{M}(s)=\frac{K_{P}}{s\left(\tau_{P} s+1\right)}
$$

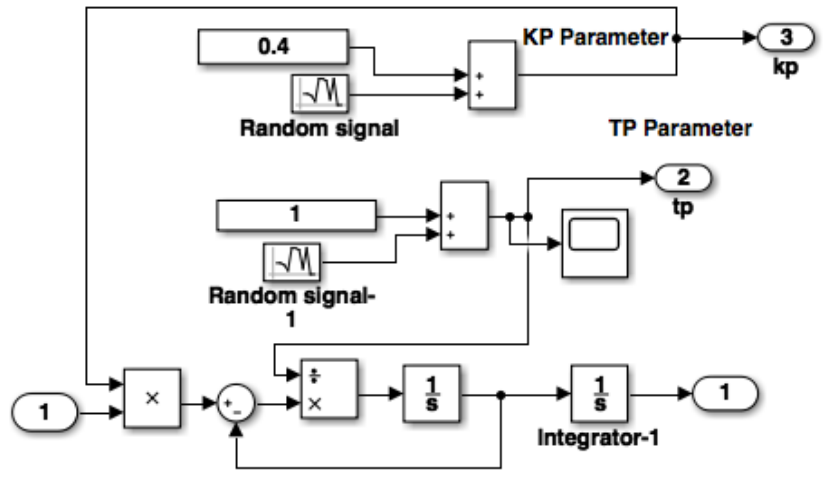

Figure 1. AC Servo Motor dynamic model Simulink block diagram

The Simulink block diagram of the AC Servo Motor dynamic model is shown in Figure 1. A random signal disturbance was injected to the system parameters $K_{P}$ and $\tau_{P}$. The values of the system parameters updated from the random signals, and then these values were passed back to the AIMC, as shown in Figure 1 and Figure 5. There were always some limitations for the motor parameter values, so the constant values were added to the random signals to keep the Motor parameter values nominal or around an average point, as shown in Table 1.

Table 1. Parameter values of AC Servo Motor [3]

\begin{tabular}{|l|l|}
\hline Type & GSM62AE \\
\hline Voltage & $230 \mathrm{~V}$ \\
\hline Moment of inertia $(\mathrm{J})$ & $0.052 \mathrm{~kg} . \mathrm{cm} 2$ \\
\hline Friction of coefficient & 0.01875 \\
\hline
\end{tabular}

\section{DESIGN OF AIMC}

To design the AIMC, first, $G_{M}(s)$ (AC Servo Motor model) of (13) was factorized into invertible and noninvertible elements [1], as shown in (14).

$$
G_{M}(s)=G_{M}(+)(s) G_{M}(-)(s)
$$

Whereby $G_{M}(+)(s)$ contained elements, whose inversion would lead to reliability and marginal stability problems as its real part was equal to zero. The assumption for the second factor of the controller $G_{M}(-)(s)$ was that its inverse leads to stability.

$$
\begin{aligned}
G_{M}(+)(s) & =\frac{1}{s} \\
G_{M}(-)(s) & =\frac{K_{P}}{\left(\tau_{P} s+1\right)}
\end{aligned}
$$

To assure that $G_{M}(+)(s)$ and $G_{M}(-)(s)$ were exclusive, the steady-state gain of the $G_{M}(+)(s)$ was required to be equal to one, and the resulting equation is shown in (17).

$$
G_{I M}(s)=G_{M}(-)(s)
$$


Subsequently, set $G_{I M}(s)$ for being inverse of $G_{M}(-)(s)$

$$
G_{M}(-)(s)^{-1}=\frac{\left(\tau_{P} s+1\right)}{K_{P}}
$$

By setting the $G_{I}(s)$ to be the inverse of the $G_{M}(-)(s)$

$$
G_{I}(s)=\frac{\left(\tau_{P} s+1\right)}{K_{P}}
$$

where

$$
G_{I M}(s)=G_{I}(s)=\frac{\left(\tau_{P} s+1\right)}{K_{P}}
$$

Since the number of zeroes was higher than the number of poles in the transfer function of the controller, the system was improper as shown in (20). To make the system proper, a Low Pass Filter was added to the system in series.

$$
G_{I M}(s)=G_{I}(s) G_{F}(s)
$$

Where

$$
G_{F}(s)=\frac{1}{\left(1+\tau_{f} s\right)^{n}}
$$

The order of the Filter ( $n$ ) was chosen as one. Increasing the order of the Filter would require more energy components, which would lead to more energy consumption. The maximum frequency component that could be handled by a system was according to its Nyquist limit, which stated that the sample rate should be higher than or equal to the double of the most significant frequency component in the input signal. According to this good rule of thumb, select the Filter parameter $\tau_{f}$ to be two times as rapid as the response of the open-loop. Hence, $\tau_{f}$ was chosen to be equal to one; this value for $\tau_{f}$ was very optimal, as increasing this value would make the system slower, and reducing this value would increase the noise in the system. Finally, the $G_{I M}(s)$ became as in (23).

$$
G_{I M}(s)=\frac{\left(\tau_{P} s+1\right)}{K_{P}} \times \frac{1}{\left(1+\tau_{f} s\right)^{n}}
$$

Substitute $\tau_{f}=1$ and $n=1$ in (23) and rearranging it, the resulting equation became as (24)

$$
G_{I M}(s)=\frac{\left(\tau_{P} s+1\right)}{K_{P} s+K_{P}}
$$

According to the internal model principle, to control the load precisely, the controller should have complete knowledge about the process that was being controlled. By comparing the motor model and the controller model, it was noted that two common parameters within the models were the system's gain parameter $K_{P}$ and the system's time parameter $\tau_{P}$ as shown below.

$$
G_{M}(s)=\frac{K_{P}}{s\left(\tau_{P} s+1\right)} \quad G_{I M}(s)=\frac{\left(\tau_{P} s+1\right)}{K_{P} s+K_{P}}
$$

Therefore, to achieve perfect control, the controller should have complete knowledge about the system's gain parameter and system's time parameter or in other words, in the controller the values of $K_{P}$ and $\tau_{P}$ should change according to the values of these parameters in the Motor model. When the dynamic load attached at the shaft of the Motor varies, the inertia of the whole system (the moment of inertia of the load $J_{L}$ and the moment of inertia of the motor $J_{M}$ ) would vary, which would change the system's time parameter $\tau_{P}$ as shown in (12). When this change occurred in the Motor model, the controller had to adapt itself accordingly.

To synchronize the Motor model and the controller a loop was needed to feed the values of $K_{P}$ and $\tau_{P}$ from the motor back to the controller. Thus, $G_{M}(s)$ and $G_{I M}(s)$ were broken into separate components and modeled in Simulink.

The controller was modeled in the Simulink by splitting it into its components and then cascading them back together.

$$
G_{I M}(s)=\frac{\left(\tau_{P} s+1\right)}{K_{P} s+K_{P}}=\frac{1}{K_{P} s+K_{P}}+\frac{\tau_{P} s}{K_{P} s+K_{P}}
$$

The first part of the controller was as shown in (26) and the second part of the controller was as shown in (27).

$$
\begin{gathered}
\frac{1}{K_{P} S+K_{P}} \\
\frac{\tau_{P} s}{K_{P} S+K_{P}}
\end{gathered}
$$

Model of the first part of the AIMC $\frac{1}{K_{P} S+K_{P}}$ is as shown in Figure 2.

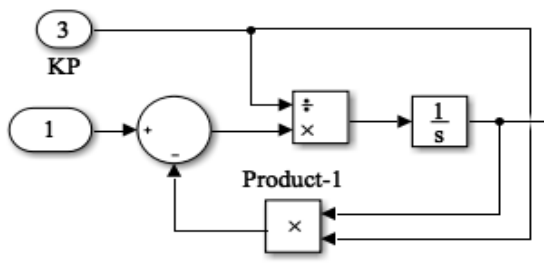

Figure 2. AIMC first part model

By combining the second part of the controller $\frac{\tau_{P} s}{K_{P} S+K_{P}}$ with the first part of the controller model, this forms the complete Simulink model of the controller transfer function $\frac{\left(\tau_{P} s+1\right)}{K_{P} s+K_{P}}$ as shown in Figure 3.

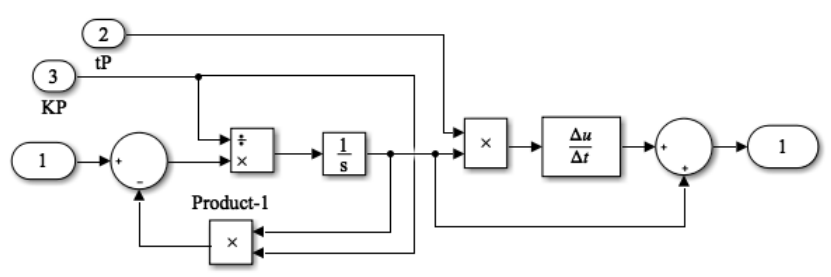

Figure 3. AIMC Simulink model 
The complete transfer function of the controller did not give the expected output because there existed some fluctuations and linearization errors. This was because the derivative block used in the model approximated the input signal with respect to the previous simulation time step and provided the approximation of $\frac{d u}{d t}$, as shown in (28). The initial output of this block was zero; it accepted an input signal and then generated an output signal [4].

$$
\frac{\Delta u}{\Delta t}=\frac{u(t)-u\left(T_{\text {Prior }}\right)}{t-T_{\text {Prior }}}
$$

Where

$t=$ current simulation time

$T_{\text {Prior }}=$ previous simulation output time

In this case, the current simulation time was always higher than the previous simulation time. Depending on the simulation time, the output signal of the system would contain unwanted fluctuations. As the derivative block output was susceptible to the entire dynamics of the model. Thus, it would affect the precision of the output signal.

\section{- Improving the performance of the dynamic system}

To do the differentiation $\left(\frac{\Delta u}{\Delta t}\right)$ in the Laplace domain, its relation would be as in (29).

$$
\frac{U s)}{X(s)}=s
$$

To approximate the linearization of the system and remove the noise from the system, a Low Pass Filter was added to the transfer function shown in (29) and (30). it would pass the low-frequency signals of the derivative function and would reject the signals whose frequencies were higher than the cutoff frequency of the Filter.

Where

$$
\frac{1}{c s+1}
$$

$$
c=\frac{1}{f_{c}}
$$

Where

$f_{c}$ is the cutoff frequency of the Filter

The location of a corner frequency was related to the frequencies at which noise occurred; it did not need to be exact as there was always some freedom in the design. Starting the corner frequency from a lower point and moving it further out until the noise was removed [5]. Following this method, it was found that to remove the noise, to achieve the best settling time and do not affect the rise time of the system, the suitable cutoff frequency required was $100 \mathrm{~Hz}$. By substituting the value of $f_{c}$ into (31).

$$
\frac{1}{0.01 s+1}
$$

To add the above Filter to the system's time parameter component in the controller model, (32) was combined with the second part of the controller (27), and the modified equation became as in (33).

$$
\frac{\tau_{P} s}{\left(K_{P} s+K_{P}\right)(0.01 s+1)}
$$

By adding back (33) to the first part of the controller (26)

$$
\frac{1}{K_{P} s+K_{P}}+\frac{\tau_{P} s}{\left(K_{P} s+K_{P}\right)(0.01 s+1)}
$$

By modifying (34), finally, the AIMC transfer function became as (35), which was modeled in Simulink, as shown in Figure 4.

$$
\frac{\left(0.01+\tau_{P}\right) s+1}{0.01 \times K_{P} s^{2}+1.01 \times K_{P} s+K_{P}}
$$

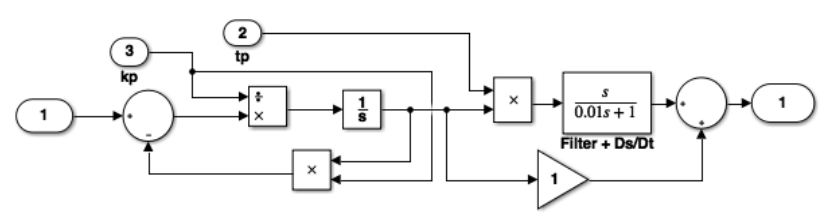

Figure 4. Final Simulink model for AIMC based on (35)

\section{DESIGN OF AIMC+FLC}

FLC was designed based on the Mamdani Min-Max method; in this Fuzzy inference system contained three stages. The first stage was fuzzification; it achieved membership degrees by the conversion of the numerical values for the use of the fuzzy mechanism. The second stage was the inference engine, which contained the fuzzy rules; it executed different fuzzy logic operations to take its actions based on the inputs. The last step was the defuzzification, which produced a crisp value from the control actions [6][16]. In the FLC design, two signals were identified, which were the error signal (Input1) and change in the error signal (Input2), as explained below.

\section{A. Input 1}

This was the error signal, it reflected the difference between the reference signal and the feedback signal, and it was used to consider the current state of the system.

\section{B. Input 2}

Input2 signal was the change in the Input1 signal. This signal identified the direction of the error variation. This signal was produced using a unit delay block and a subtraction block, in which the signal was delayed by a sample time of 0.1 seconds, and then it was subtracted from the Input1 signal, which resulted in the difference between the error signal and delayed signal [6]. The sampling time (36) was chosen based on [6] [7] [8].

$$
T_{\text {Sampling }} \leq 0.1 \times T_{\text {Process }}
$$

$T_{\text {Process }}$ was the processing time constant. This was defined as the difference between the time at which process variable showed its first response to the control output step and the time at which process variable reached up to $63 \%$ of the total process variable change or the final steady-state value. Here the processing time was 
chosen based on the AIMC response, which was 1.48 seconds. By substituting the process time value in (36), as shown in (37).

$$
T_{\text {Sampling }} \leq 0.148 \text { seconds }
$$

It was observed that the system led to the best response at 0.1 seconds of sampling time.

The two signals, as explained above (Input1 and Input2), were passed to Multiplexer (Mux), which combined these two signals graphically and generated a single vector output signal. The order of the elements in the vector output signal was taken from top to bottom. The output signal of the Mux was then passed to FLC, which implemented the FIS. The Simulink model of the FLC connected with the AIMC is shown in Figure 5. The FLC settings are shown in Figure 6.

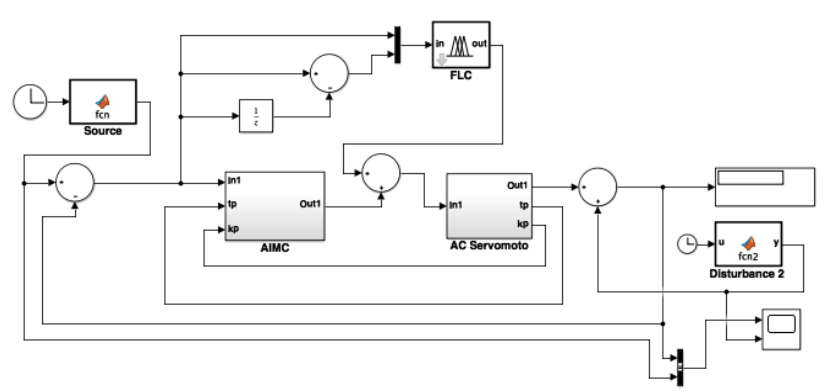

Figure 5. Simulink model of AIMC+FLC

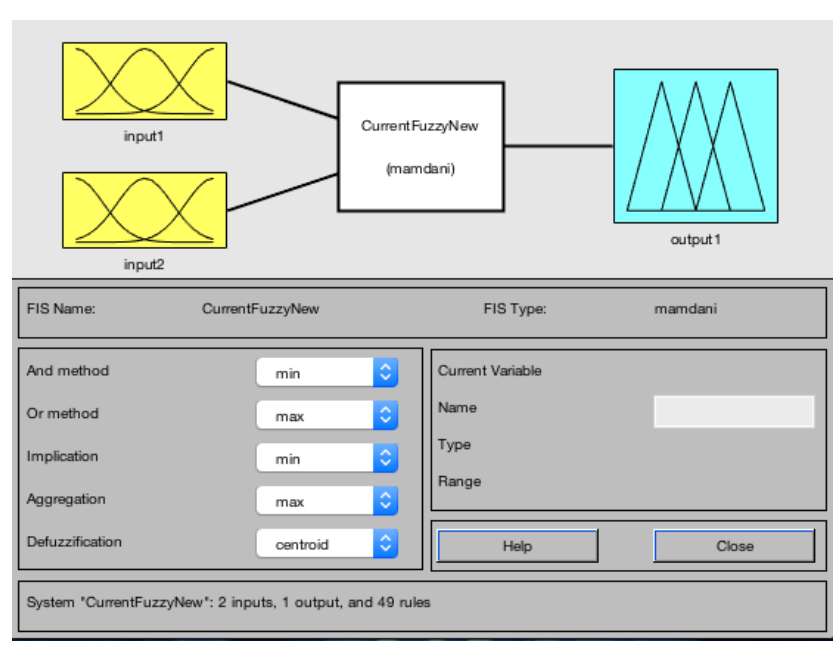

Figure 6. FLC Settings

\section{FLC Rules}

The rules of the FLC were implemented based on the Fuzzy Implication Rules, i.e., IF-THEN rules, as shown in Table 2. AND (Min) operator was used between the two variables Input1 and Input2. The fuzzy output was achieved by the combination of the different productions of the rules utilizing OR (Max) operator. These control rules stated that the FLC rules could be implemented based on the general logic between the input and output waveforms using the linguistic variables [17].
Table 2. FLC rules

\begin{tabular}{|c|c|c|c|c|c|c|c|}
\hline Input 1 & \multirow{2}{*}{ NB } & NM & NS & ZE & PS & PM & PB \\
\hline NB & NB & NB & NB & NB & NM & NS & ZE \\
\hline NM & NB & NB & NB & NM & NS & ZE & PS \\
\hline NS & NB & NB & NM & NS & ZE & PS & PM \\
\hline ZE & NB & NM & NS & ZE & PS & PM & PB \\
\hline PS & NM & NS & ZE & PS & PM & PB & PB \\
\hline PM & NS & ZE & PS & PM & PB & PB & PB \\
\hline PB & ZE & PS & PM & PB & PB & PB & PB \\
\hline
\end{tabular}

\section{RESULT}

\subsection{Results of IMC}

The IMC was not able to control a dynamic load precisely when the load would change; the inertia of the load would vary, which would cause the system's time parameter to change. Such variations would affect the precision of the controller. This occurred because the controller only worked based on the given information of the static load, and when the load changed, the controller could not absorb the latest information of the load such that it could not withstand itself with the new characteristics of the load. Assuming that a dynamic load was attached at the shaft of the AC Servo Motor, as shown in Figure 7. Thus, the new values of the load inertia and load friction became as

$$
\begin{gathered}
J_{L}=0.15 \mathrm{Kg} \cdot \mathrm{m}^{2} \\
B_{L}=0.001
\end{gathered}
$$

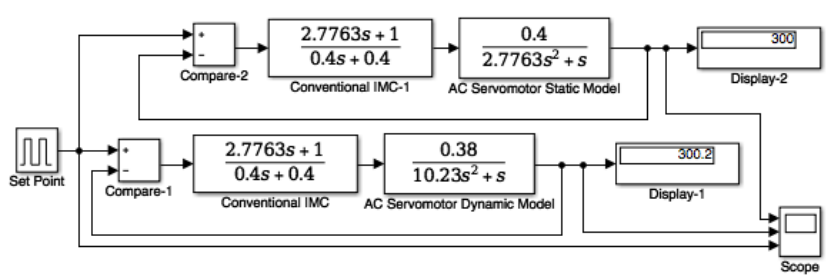

Figure 7. Simulink model of IMC under static and dynamic load conditions

Figure 8 shows the comparison between the output waveforms of the IMC under static and dynamic load conditions.

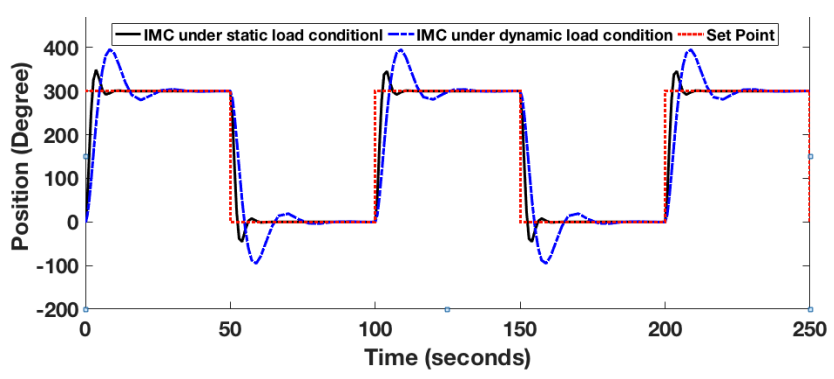

Figure 8. IMC performance under static and dynamic load conditions

By varying the load inertia by a small value such as choosing $J_{L}=0.2 \mathrm{Kg} \cdot \mathrm{m}^{2}$ the performance of the controller became even worse as shown in Figure 9. 


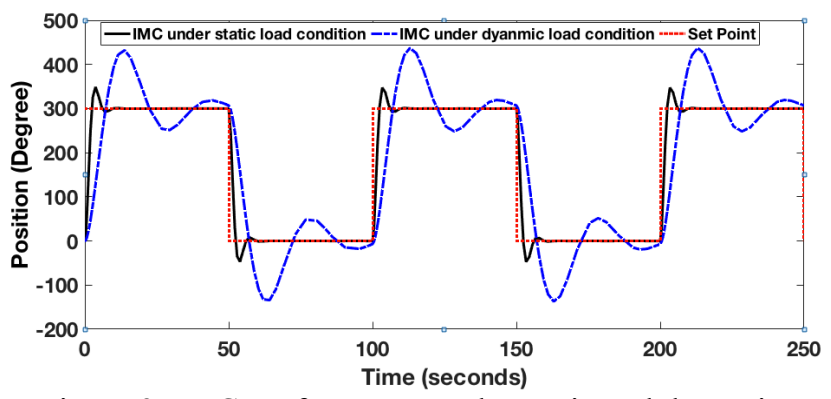

Figure 9. IMC Performance under static and dynamic load conditions (with higher change in the load)

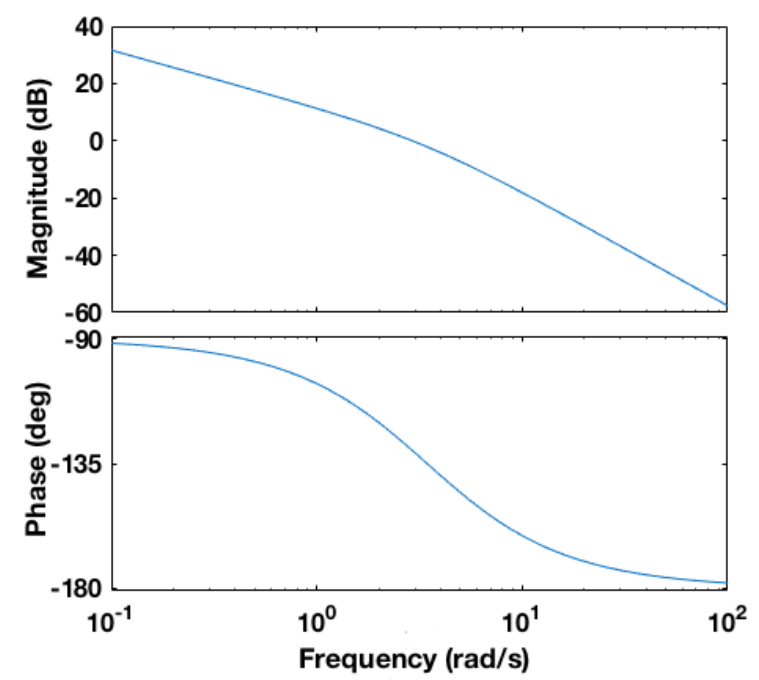

Figure 10. Bode diagram of AC Servo Motor at static load condition under IMC

Figure 10 shows the bode diagram of AC Servo Motor under static load conditions. The system was found stable under static load conditions. When a dynamic load was connected to the IMC, its stability started reducing, the phase margin of the plot was reduced to $9.12^{\circ}$ and its delay time margin was reduced to 0.0188 seconds. This indicated that the stability of the system reduced with respect to the new load conditions, as shown in Figure 11.
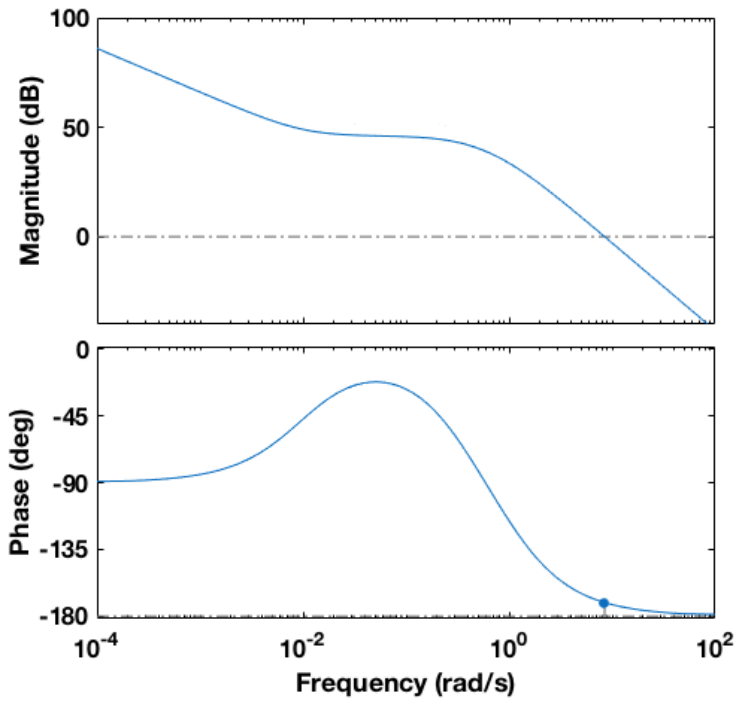

Figure 11. Bode plot of IMC under dynamic load condition

\subsection{Robustness of IMC again external disturbances}

It could be seen from Figure 13 and Figure 14 that by injecting disturbance of an amplitude of 10 at the output of the Motor, as shown in Figure 12. The IMC lost control on the AC Servo Motor and Motor. This was due to the difference in the motor time parameter values.

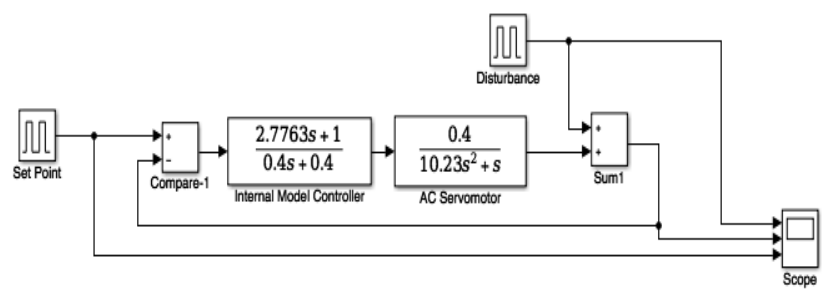

Figure 12. Simulink model of IMC under dynamic load condition with disturbance injection

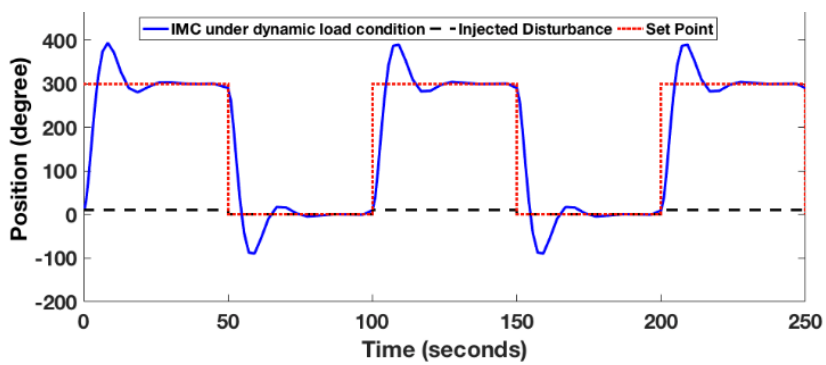

Figure 13. AC Servo Motor under dynamic load condition when controlled by IMC and disturbance injected at the output of the Motor

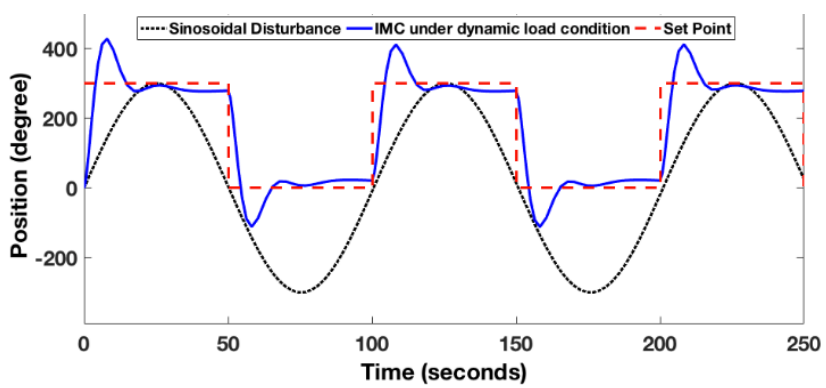

Figure 14. AC Servo Motor under dynamic load condition when controlled by IMC and Sinusoidal disturbance injected at the output of the Motor

\subsection{Results of AIMC}

Figure 15 shows the setpoint tracking performance of the AIMC and IMC. It was noted that the tracking performances of these two controllers were similar. The step response characteristics of these two systems are compared in Table 3.

Figure 16 shows the bode diagram of the AC Servo Motor with AIMC under dynamic load conditions. From the stability analysis, it could be seen that the phase margin of the AIMC was higher than the IMC, and its delay margin was less than the IMC, as shown in Table 4. The stability of the system could be improved by a larger phase and gain margins [9]. For a given operating point, the maximum amount of time delay that a system could tolerate before it would become unstable was the time delay margin. The time delay margin of AIMC was less than the IMC, but it was still an acceptable value for the robustness of the system. 


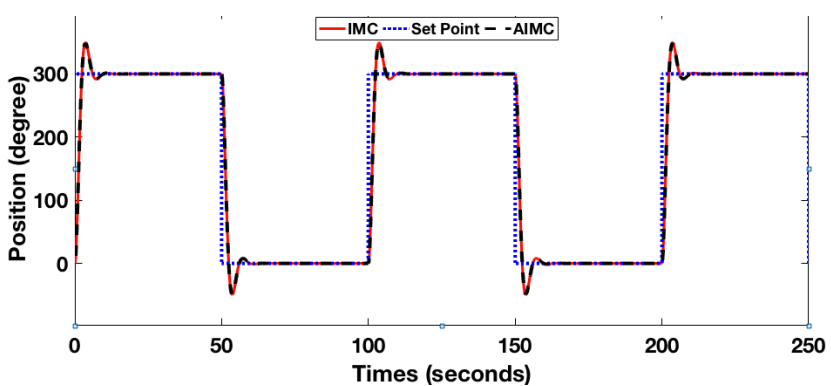

Figure 15. Setpoint tracking performance of the AIMC and IMC

Table I3. Comparison between the step response of AIMC and IMC

\begin{tabular}{|l|c|c|}
\hline $\begin{array}{l}\text { Response } \\
(\text { State Level Tolerance =2\%) }\end{array}$ & AIMC & IMC \\
\hline Overshoot (\%) & 16.66 & 16.666 \\
\hline Undershoot (\%) & 2.805 & 2.397 \\
\hline Settling Time (seconds) & 8.05 & 8.13 \\
\hline Rise Time (seconds) & 1.625 & 1.626 \\
\hline
\end{tabular}

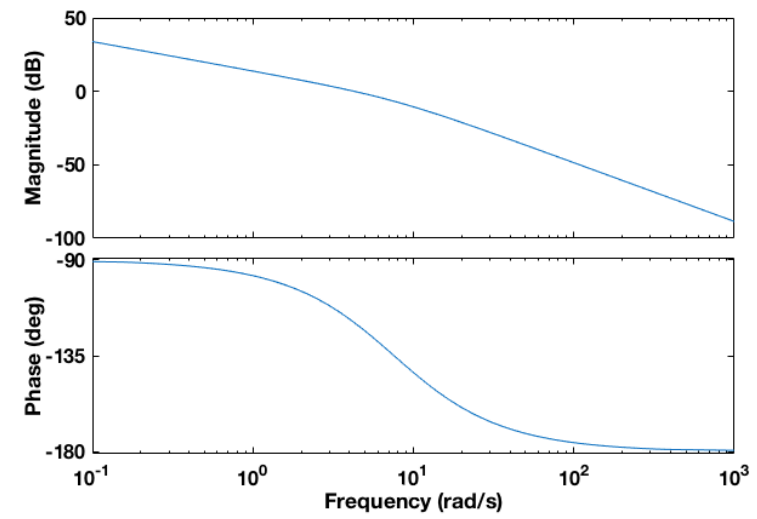

Figure 16. Bode diagram of AC Servo Motor at dynamic load condition under AIMC

Table 4. Stability comparison of AC Servo Motor under AIMC and IMC

\begin{tabular}{|l|c|c|}
\hline Stability Characteristics & AIMC & IMC \\
\hline Stable & Yes & Yes \\
\hline Phase Margin (degree) & $60.5^{\circ}$ & $49.7^{\circ}$ \\
\hline Time Delay Margin (seconds) & 2.35 & 2.84 \\
\hline
\end{tabular}

\subsection{Robustness of AIMC against external disturbances}

Different types of waveforms were injected to observe the robustness of the controller against external disturbances at the output of the Motor as shown in Figure 17, Figure 18, Figure 19, and Figure 20

From Figure 19 and Figure 20, it could be seen that the Motor response under AIMC was not affected significantly when a sinusoidal disturbance of smaller amplitude was injected at the output of the Motor. The disturbance effects increased with an increase in the magnitude of the waveform. AIMC+FLC improved the solution of this issue.

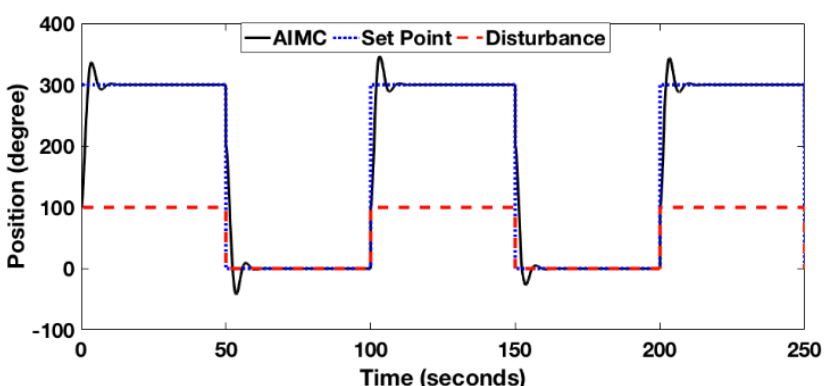

Figure 17. AC Servo Motor response under AIMC when disturbance with an amplitude of 100 was injected at the output of the Motor

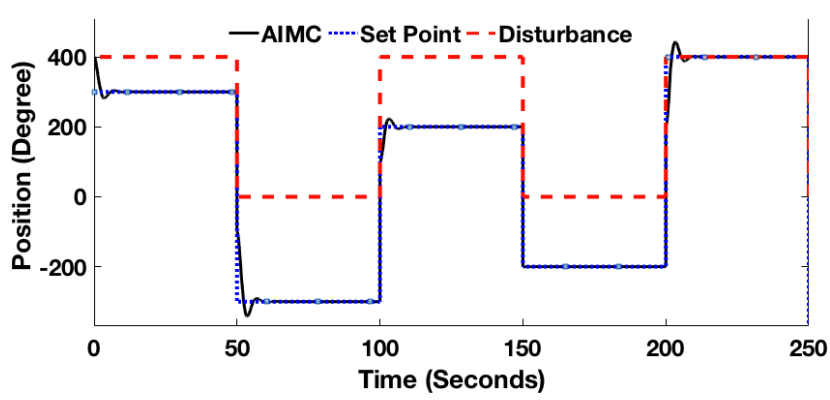

Figure 18. AC Servo Motor response under AIMC when disturbance with an amplitude of 400 was injected at the output of the Motor

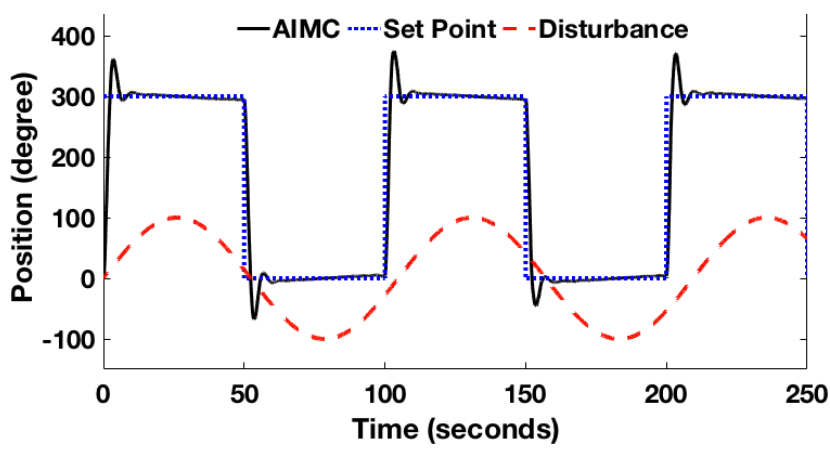

Figure 19. AC Servo Motor response under AIMC when a sinusoidal disturbance with an amplitude of 100 was injected at the output of the Motor

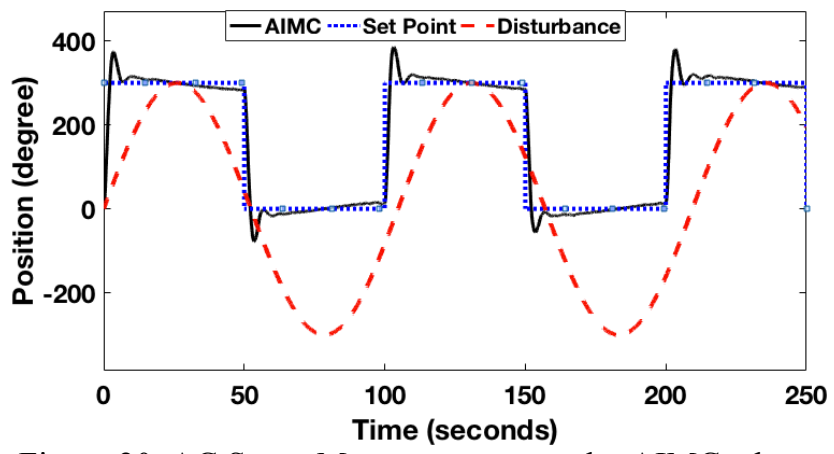

Figure 20. AC Servo Motor response under AIMC when a sinusoidal disturbance with an amplitude of 300 was injected at the output of the Motor 


\subsection{Results of AIMC+FLC}

The step response of the system was improved with a massive difference by connecting FLC with the AIMC, as shown in Figure 21.

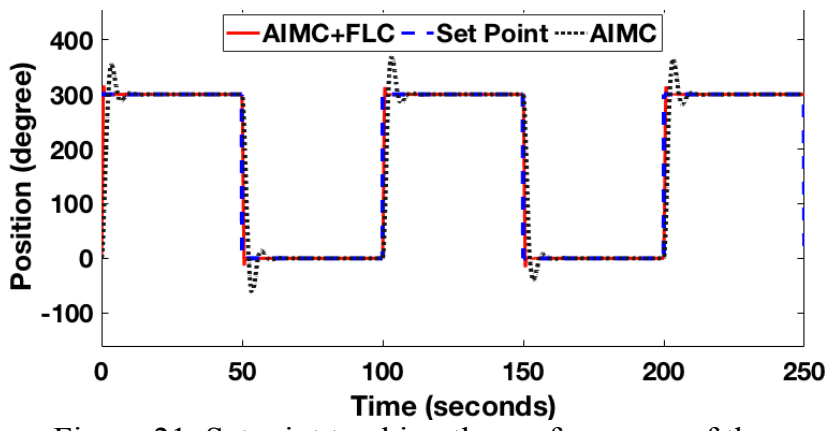

Figure 21. Setpoint tracking the performance of the Motor under AIMC+FLC

The step response comparison between the three controllers is shown in Table 5.

Table 5. Comparison between the step responses of FLC+AIMC, AIMC and IMC

\begin{tabular}{|l|c|c|c|}
\hline $\begin{array}{l}\text { Response (State Level } \\
\text { Tolerance = 2\%) }\end{array}$ & $\begin{array}{c}\text { AIMC+ } \\
\text { FLC }\end{array}$ & AIMC & IMC \\
\hline Overshoot (\%) & 4.411 & 16.66 & 16.666 \\
\hline Undershoot (\%) & 1.944 & 2.805 & 2.397 \\
\hline Settling Time (seconds) & 0.6 & 8.05 & 8.13 \\
\hline Rise Time (seconds) & 0.429 & 1.625 & 1.626 \\
\hline
\end{tabular}

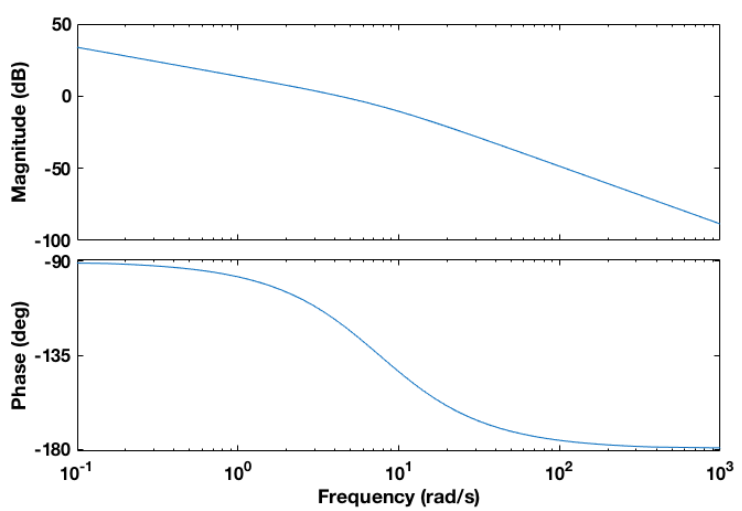

Figure 22. Bode plot of AC Servo Motor under AIMC+FLC

The phase margin and delay margin of the AIMC+FLC was found stable, as shown in Figure 22. Its stability characteristics were compared with the AIMC and IMC in Table 6. The phase margin and delay margin of the AIMC+FLC were less compared to the other two controllers, but they were still acceptable, and the system was well stable.

Table 6. Stability analysis of the AIMC+FLC, AIMC, and IMC

\begin{tabular}{|l|c|c|c|}
\hline Stability Characteristics & $\begin{array}{c}\text { AIMC+ } \\
\text { FLC }\end{array}$ & AIMC & IMC \\
\hline Stable & Yes & Yes & Yes \\
\hline Phase Margin (degree) & $43.1^{\circ}$ & $60.5^{\circ}$ & $49.7^{\circ}$ \\
\hline Time Delay Margin (seconds) & 2.13 & 2.35 & 2.84 \\
\hline
\end{tabular}

5.6 Robustness of AIMC+FLC against external disturbances

The robustness of AIMC+FLC against external interference was further observed in Figure 23, Figure 24, Figure 25, and Figure 26 by injecting various types of disturbances at the output of the Motor.

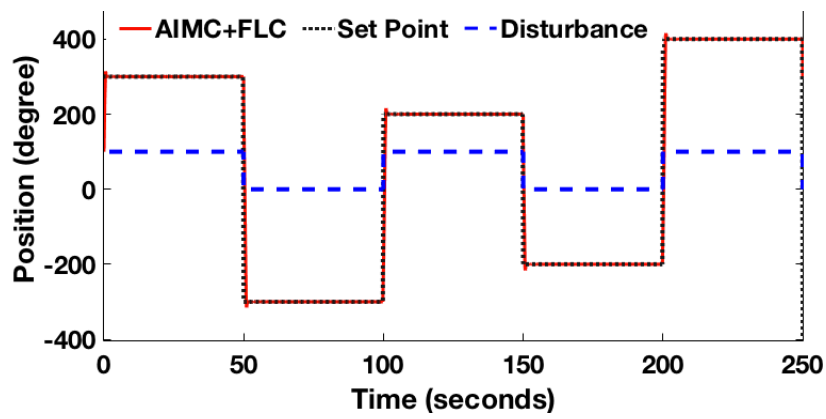

Figure 23. AC Servo Motor response under AIMC+FLC when disturbance with an amplitude of 100 was injected at the output of the Motor

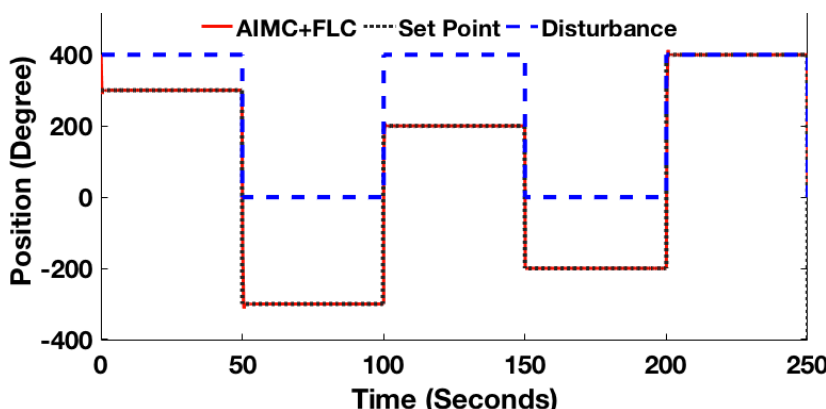

Figure 24. AC Servo Motor response under AIMC+FLC when disturbance with an amplitude of 400 was injected at the output of the Motor

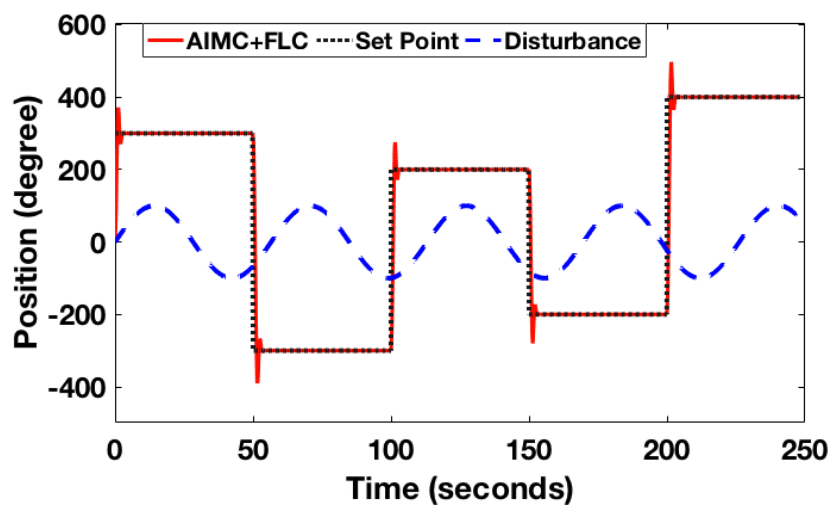

Figure 25. AC Servo Motor response under AIMC+FLC when a disturbance of higher frequency Sinusoidal waveform with an amplitude of 100 was injected at the output of the Motor

\section{CONCLUSION}

In this paper, the IMC technique was further improved for the precise control of the dynamic loads, and for the robustness against external disturbances, the proposed control technique is AIMC. The AIMC is designed and creatively modeled in the Simulink; its simulation results were compared with the IMC and further analyzed. The results indicated that AIMC could precisely control the 
position of the AC Servo Motor under dynamic load conditions, and it was more robust against external disturbances.

The step response and the robustness of the AIMC against external disturbances were further improved by designing a FLC and connecting it with the AIMC and the proposed controller is AIMC+FLC. It was observed that the AIMC+FLC step response and its robustness against external disturbances were much better than the AIMC and IMC.

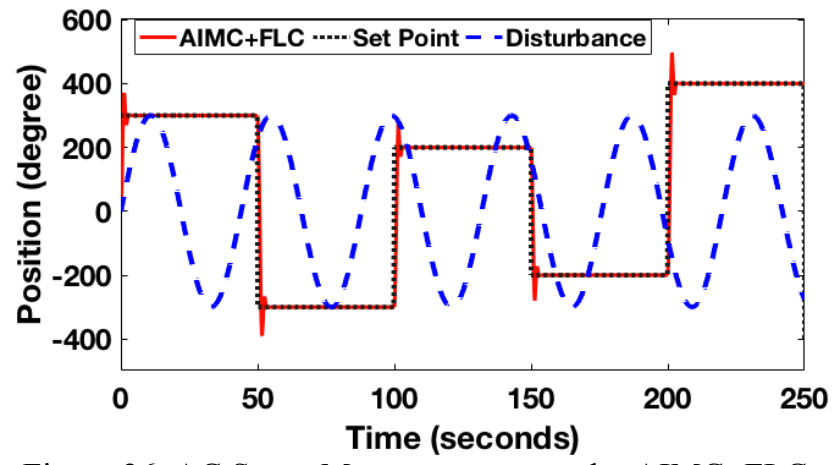

Figure 26. AC Servo Motor response under AIMC+FLC when a disturbance of higher frequency Sinusoidal waveform with an amplitude of 300 was injected at the output of the Motor

\section{ACKNOWLEDGMENT}

This study was supported by faculty of Engineering, Multimedia University, Malaysia.

\section{REFERENCES}

[1] S. A. El-Hamid And A. H. Eissa, "Position Control Of Ac Servo Motor Using Internal Model Control Strategy," Int. J. Eng. Innov. Res., Vol. 4, No. 2, Pp. 277-281, 2015.

[2] Makes And T. Fun, "Ac Servo Motor", Ls Mecapion.

[3] M. Vijayakarthick And P.K. Bahaba, "Position Tracking Performance Of Ac Servo Motor Based On New Modified Repetitive Control Strategy," Vol. 10, No. January, Pp. 119-128, 2012.

[4] "Output Time Derivative Of Input - Mathworks Simulink."

[5] K. Lacanette, "A Basic Introduction To FiltersActive, Passive, And Switched-Capacitor," In Nat. Semiconductor Corporation Applicant, 2010, Pp. 118.
[6] B. Shikkewal And V. Nandanwar, "Fuzzy Logic Controller For Pmsm," Int. J. Electr. Electron. Eng., vol. 1, no. 3, pp. 73-78, 2012

[7] M. Negnevitsky, "Fuzzy expert systems," Artif. Intell. A Guid. To Intell. Syst., pp. 87-130, 2011.

[8] "Sample Time is a Fundamental Design and Tuning Specification - Practical Process Control by Control Guru." 2007.

[9] L. H. Keel and S. P. Bhattacharyya, "A Bode Plot Characterization of All Stabilizing Controllers," IEEE Trans. Automat. Contr., vol. 55, no. 11, pp. 2650-2654, Nov. 2010.

[10] S. P. Singh, A. K. Gautam, J. Dubey, J. P. Pandey, and R. P. Payasi, "Performance Comparison of PMSM Drive using PI and Fuzzy Logic based controllers," pp. 563-569, 2016.

[11] F. Information, "Fuzzy Control Strategies in Human Operator and Sport Modeling Fuzzy Control Strategies in Human Operator and Sport Modeling," no. July 2009, 2014.

[12] F. F. Cheng and S. N. Yeh, "Application of fuzzy logic in the speed control of AC servo system and an intelligent inverter," IEEE Trans. Energy Convers., vol. 8, no. 2, pp. 312-318, Jun. 1993.

[13] J. Yin, L. Liu, and S. Shi, "Fuzzy Adaptive PID Controlling of Servo Motor System Based on DSP," Fifth Int. Conf. Intell. Human-Mach. Syst. Cybern., pp. 26-28, 2013.

[14] S. Wang, W. Zhu, J. Shi, H. J, and S. Huang, "A High Performance Permanent Magnet Synchronous Motor Servo System Using Predictive Functional Control and Kalman Filter," J. Power Electron., vol. 15, no. 6, pp. 1547-1558, Nov. 2015.

[15] F. Authors, "COMPEL: The International Journal for Computation and Mathematics in Electrical and Electronic Engineering Article information :," 2015.

[16] Sheikhidris, K., Alias, M. Y., \& Roslee, M. (2017, November). Adaptive power management with fractional frequency reuse scheme for co-tier Femto-cell interference reduction. In 2017 IEEE 13th Malaysia International Conference on Communications (MICC) (pp. 311-315). IEEE.

[17] Sheikhidris, K., Alias, M. Y., Roslee, M., \& Alam, M. J. (2018, May). Throughput Maximization Based on User Association in Heterogeneous Networks. In 2018 6th International Conference on Information and Communication Technology (ICoICT) (pp. 34-37). IEEE. 